\title{
Binary Bargmann Symmetry Constraints of Soliton Equations
}

\author{
Wen-Xiu Ma \\ Department of Mathematics, City University of Hong Kong, Hong Kong, \\ Kowloon, China
}

\begin{abstract}
Binary Bargmann symmetry constraints are applied to decompose soliton equations into finite-dimensional Liouville integrable Hamiltonian systems, generated from so-called constrained flows. The resulting constraints on the potentials of soliton equations give rise to involutive solutions to soliton equations, and thus the integrability by quadratures are shown for soliton equations by the constrained flows. The multi-wave interaction equations associated with the $3 \times 3$ matrix AKNS spectral problem are chosen as an illustrative example to carry out binary Bargmann symmetry constraints. The Lax representations and the corresponding $\mathbf{r}$-matrix formulation are established for the constrained flows of the multi-wave interaction equations, and the integrals of motion generated from the Lax representations are utilized to show the Liouville integrability for the resulting constrained flows. Finally, involutive solutions to the multi-wave interaction equations are presented.
\end{abstract}

Key words:

Symmetry constraints, Binary nonlinearization, Liouville integrability

PACS: 02.30.Jr - Partial differential equations.

\section{Introduction}

The nonlinearization technique has been attracting broad attention in the soliton community [1-3]. It provides a powerful approach for analyzing soliton equations, in both continuous and discrete cases, especially for showing the integrability by quadratures for soliton equations. A kind of specific Bargmann symmetry constraints [4] yields the required constraints on the potentials of soliton equations in nonlinearization. One of two conserved covariants involved in each Bargmann symmetry constraint is Lie point, but the other is not Lie point, contact or Lie-Bäcklund, which is expressed in terms of eigenfunctions 
and adjoint eigenfunctions of spectral problems associated with soliton equations. Such symmetry constraints nonlinearize spectral problems of soliton equations into finite-dimensional Liouville integrable systems, and the constraints on the potentials present involutive solutions of soliton equations.

Binary Bargmann symmetry constraints also establish a bridge between infinite-dimensional soliton equations and finite-dimensional Liouville integrable systems [4]. The constrained flows resulted from spectral problems of soliton equations are utilized to show the integrability by quadratures for soliton equations $[5,6]$ and pave a method of separation of variables for soliton equations $[7,8]$. The study of binary symmetry constraints itself is an important part of the kernel of the mathematical theory of nonlinearization.

This paper is structured as follows. In section 2, a general theory is recalled for reference. Then starting from section 3, an illustrative example is carried out to manipulate binary Bargmann symmetry constraints. Finally in section 8, a summary is given along with concluding remarks.

\section{General theory}

Let us address a skeleton theory of binary Bargmann symmetry constraints (for example, see [9] for details). We start from a matrix spectral problem

$$
\phi_{x}=U \phi=U(u, \lambda) \phi, U=\left(U_{i j}\right)_{r \times r}, \phi=\left(\phi_{1}, \cdots, \phi_{r}\right)^{T}
$$

with a spectral parameter $\lambda$ and a potential $u=\left(u_{1}, \cdots, u_{q}\right)^{T}$. Suppose that the compatability conditions $U_{t_{m}}-V_{x}^{(m)}+\left[U, V^{(m)}\right]=0, m \geq 0$, of the spectral problem (2.1) and the associated spectral problems

$$
\phi_{t_{m}}=V^{(m)} \phi=V^{(m)}\left(u, u_{x}, \cdots ; \lambda\right) \phi, V^{(m)}=\left(V_{i j}^{(m)}\right)_{r \times r}, m \geq 0
$$

determine an isospectral $\left(\lambda_{t_{m}}=0\right)$ soliton hierarchy

$$
u_{t_{m}}=X_{m}(u)=J G_{m}=J \frac{\delta \tilde{H}_{m}}{\delta u}, m \geq 0,
$$

where the Hamiltonian operator $J$ and the Hamiltonian functionals $\tilde{H}_{m}$ can be determined by a trace identity. Evidently, the compatability conditions of the adjoint spectral problem and the adjoint associated spectral problems

$$
\psi_{x}=-U^{T}(u, \lambda) \psi, \psi_{t_{m}}=-V^{(m) T}(u, \lambda) \psi, m \geq 0,
$$


where $\psi=\left(\psi_{1}, \cdots, \psi_{r}\right)^{T}$, still give rise to the same soliton hierarchy $(2.3)$.

It has been pointed out [4] that $J \frac{\delta \lambda}{\delta u}=E^{-1} J \psi^{T} \frac{\partial U(u, \lambda)}{\partial u} \phi$ is a common symmetry of all equations in the hierarchy (2.3), where $E$ is the normalized constant. Upon introducing $N$ distinct eigenvalues $\lambda_{1}, \cdots, \lambda_{N}$, we obtain

$$
\begin{aligned}
& \phi_{x}^{(s)}=U\left(u, \lambda_{s}\right) \phi^{(s)}, \psi_{x}^{(s)}=-U^{T}\left(u, \lambda_{s}\right) \psi^{(s)}, 1 \leq s \leq N \\
& \phi_{t_{m}}^{(s)}=V^{(m)}\left(u, \lambda_{s}\right) \phi^{(s)}, \psi_{t_{m}}^{(s)}=-V^{(m) T}\left(u, \lambda_{s}\right) \psi^{(s)}, 1 \leq s \leq N,
\end{aligned}
$$

where the corresponding eigenfunctions and adjoint eigenfunctions are denoted by $\phi^{(s)}$ and $\psi^{(s)}, 1 \leq s \leq N$. It is assumed that the conserved covariant $G_{m_{0}}$ is Lie point, and then the so-called binary Bargmann symmetry constraint reads

$$
X_{m_{0}}=\sum_{s=1}^{N} E_{s} J \frac{\delta \lambda_{s}}{\delta u} \text {, i.e., } J G_{m_{0}}=J \sum_{s=1}^{N} \psi^{(s) T} \frac{\partial U\left(u, \lambda_{s}\right)}{\partial u} \phi^{(s)},
$$

where $E_{s}, 1 \leq s \leq N$, are $N$ normalized constants. The right-hand side of the symmetry constraint (2.7) is a linear combination of $N$ symmetries $E_{s} J \delta \lambda_{s} / \delta u, 1 \leq s \leq N$. Such symmetries (or the corresponding conserved covariants $\left.\delta \lambda_{s} / \delta u, 1 \leq s \leq N\right)$ are not Lie point, contact or Lie-Bäcklund symmetries, since $\phi^{(s)}$ and $\psi^{(s)}$ can not be expressed in terms of $x, u$ and derivatives of $u$ with respect to $x$ to some finite order.

Suppose that the symmetry constraint (2.7) has an inverse function

$$
u=\widetilde{u}\left(\phi^{(1)}, \cdots, \phi^{(N)} ; \psi^{(1)}, \cdots, \psi^{(N)}\right) .
$$

Replacing $u$ with $\widetilde{u}$ in the system (2.5) or the system (2.6), we obtain the so-called spatial constrained flow and the so-called temporal constrained flow:

$$
\begin{gathered}
\phi_{x}^{(s)}=U\left(\widetilde{u}, \lambda_{s}\right) \phi^{(s)}, \psi_{x}^{(s)}=-U^{T}\left(\widetilde{u}, \lambda_{s}\right) \psi^{(s)}, 1 \leq s \leq N \\
\phi_{t_{m}}^{(s)}=V^{(m)}\left(\widetilde{u}, \lambda_{s}\right) \phi^{(s)}, \psi_{t_{m}}^{(s)}=-V^{(m) T}\left(\widetilde{u}, \lambda_{s}\right) \psi^{(s)}, 1 \leq s \leq N .
\end{gathered}
$$

The former is a system of ordinary differential equations, and the latter is usually a system of partial differential equations but it can be transformed into a system of ordinary differential equations under the control of (2.9).

The main question of symmetry constraints is to show that the spatial constrained flow (2.9) and the temporal constrained flows (2.10) under the control of (2.9) are Liouville integrable. Then if $\phi^{(s)}$ and $\psi^{(s)}, 1 \leq s \leq N$, solve two 
constrained flows (2.9) and (2.10) simultaneously, $u=\widetilde{u}$ will present a solution to the $m$ th soliton equation $u_{t_{m}}=X_{m}(u)$. It also follows that the soliton equation $u_{t_{m}}=X_{m}(u)$ is decomposed into two finite-dimensional Liouville integrable systems, and the constraint $u=\widetilde{u}$ gives a Bäcklund transformation between soliton equations and the resulting finite-dimensional Liouville integrable systems [10]. The whole process to carry out binary Bargmann symmetry constraints is called binary nonlinearization $[11,5]$.

\section{Multiwave interaction equations}

Let us begin with the $3 \times 3$ matrix AKNS spectral problem:

$$
\phi_{x}=U(u, \lambda) \phi, U=\left(\begin{array}{ccc}
\alpha_{1} \lambda & u_{12} & u_{13} \\
u_{21} & \alpha_{2} \lambda & u_{23} \\
u_{31} & u_{32} & \alpha_{3} \lambda
\end{array}\right)=\lambda U_{0}+U_{1}, \phi=\left(\begin{array}{l}
\phi_{1} \\
\phi_{2} \\
\phi_{3}
\end{array}\right)
$$

where $U_{0}=\operatorname{diag}\left(\alpha_{1}, \alpha_{2}, \alpha_{3}\right)$, and $\alpha_{1}, \alpha_{2}, \alpha_{3}$ are distinct constants, and the potential $u$ is defined by

$$
u=\rho\left(U_{1}\right)=\left(u_{21}, u_{12}, u_{31}, u_{13}, u_{32}, u_{23}\right)^{T}
$$

We introduce an associated spectral problem:

$$
\phi_{t}=V(u, \lambda) \phi, V=\left(\begin{array}{ccc}
\beta_{1} \lambda & v_{12} & v_{13} \\
v_{21} & \beta_{2} \lambda & v_{23} \\
v_{31} & v_{32} & \beta_{3} \lambda
\end{array}\right)=\lambda V_{0}+V_{1}
$$

where $V_{0}=\operatorname{diag}\left(\beta_{1}, \beta_{2}, \beta_{3}\right)$, and $\beta_{1}, \beta_{2}, \beta_{3}$ are distinct constants. Under the isospectral condition $\lambda_{t}=0$, the compatibility condition $U_{t}-V_{x}+[U, V]=0$ is equivalent to

$$
U_{1 t}-V_{1 x}+\left[U_{1}, V_{1}\right]=0,\left[U_{0}, V_{1}\right]=\left[V_{0}, U_{1}\right]
$$

They give rise to the multi-wave interaction equations

$$
u_{i j, t}=\frac{\beta_{i}-\beta_{j}}{\alpha_{i}-\alpha_{j}} u_{i j, x}+\sum_{\substack{k=1 \\ k \neq i, j}}^{3}\left(\frac{\beta_{k}-\beta_{i}}{\alpha_{k}-\alpha_{i}}-\frac{\beta_{k}-\beta_{j}}{\alpha_{k}-\alpha_{j}}\right) u_{i k} u_{k j}, \quad 1 \leq i \neq j \leq 3
$$


which contain three-wave interaction equations arising in fluid dynamics and plasma physics $[12,13]$, with $U_{1}$ being chosen to be an anti-Hermitian matrix. Note that the compatability condition of the adjoint spectral problem and adjoint associated spectral problem:

$$
\psi_{x}=-U^{T}(u, \lambda) \psi, \psi_{t}=-V^{T}(u, \lambda) \psi, \psi=\left(\psi_{1}, \psi_{2}, \psi_{3}\right)^{T}
$$

still gives rise to the above multi-wave interaction equations. It is easy to find

that the multi-wave interaction equations (3.4) have the Hamiltonian structure

$$
u_{i j, t}=J \frac{\delta \tilde{H}}{\delta u_{i j}}, \quad 1 \leq i \neq j \leq 3
$$

where the Hamiltonian operator $J$ and the Hamiltonian $\tilde{H}$ are defined by

$$
\begin{aligned}
J & =\operatorname{diag}\left(\left(\alpha_{1}-\alpha_{2}\right) \sigma_{0},\left(\alpha_{1}-\alpha_{3}\right) \sigma_{0},\left(\alpha_{2}-\alpha_{3}\right) \sigma_{0}\right), \sigma_{0}=\left(\begin{array}{cc}
0 & 1 \\
-1 & 0
\end{array}\right), \\
\tilde{H} & =\int H d x, H=\frac{1}{3\left(\alpha_{1}-\alpha_{2}\right)}\left(\frac{\beta_{3}-\beta_{2}}{\alpha_{3}-\alpha_{2}}-\frac{\beta_{3}-\beta_{1}}{\alpha_{3}-\alpha_{1}}\right)\left(u_{12} u_{23} u_{31}\right. \\
& \left.+u_{21} u_{13} u_{32}\right)+\frac{\beta_{1}-\beta_{2}}{2\left(\alpha_{1}-\alpha_{2}\right)^{2}}\left(u_{12} u_{21, x}-u_{21} u_{12, x}\right)+\operatorname{cycle}(1,2,3)
\end{aligned}
$$

\section{Binary Bargmann symmetry constraints}

Let us consider binary Lax systems of the multi-wave interaction equations $(3.4)$ :

$$
\begin{gathered}
\phi_{x}=U(u, \lambda) \phi, \psi_{x}=-U^{T}(u, \lambda) \psi ; \\
\phi_{t}=V(u, \lambda) \phi, \psi_{t}=-V^{T}(u, \lambda) \psi .
\end{gathered}
$$

Based on the Hamiltonian theory, the conserved functional $\lambda(u)$ gives rise to a symmetry of (3.4):

$$
E J \frac{\delta \lambda}{\delta u}=E \rho\left(\left[U_{0}, \rho^{-1}\left(\frac{\delta \lambda}{\delta u}\right)\right]\right)=\rho\left(\left[U_{0}, \rho^{-1}\left(\psi^{T} \frac{\partial U(u, \lambda)}{\partial u} \phi\right)\right]\right)
$$


where $E$ is the normalized constant and $\rho$ is the mapping defined by (3.2). Having introduced $N$ distinct eigenvalues $\lambda_{1}, \cdots, \lambda_{N}$, we obtain

$$
\begin{aligned}
& \phi_{x}^{(s)}=U\left(u, \lambda_{s}\right) \phi^{(s)}, \psi_{x}^{(s)}=-U^{T}\left(u, \lambda_{s}\right) \psi^{(s)}, 1 \leq s \leq N \\
& \phi_{t}^{(s)}=V\left(u, \lambda_{s}\right) \phi^{(s)}, \psi_{t}^{(s)}=-V^{T}\left(u, \lambda_{s}\right) \psi^{(s)}, 1 \leq s \leq N
\end{aligned}
$$

where $\phi^{(s)}$ and $\psi^{(s)}$ are assumed to be denoted by

$$
\phi^{(s)}=\left(\phi_{1 s}, \phi_{2 s}, \phi_{3 s}\right)^{T}, \psi^{(s)}=\left(\psi_{1 s}, \psi_{2 s}, \psi_{3 s}\right)^{T}, 1 \leq s \leq N .
$$

This multi-eigenvalue case yields a general symmetry of (3.4) (not Lie point, contact or Lie Bäcklund type):

$$
Z_{0}:=\rho\left(\left[U_{0}, \rho^{-1}\left(\sum_{s=1}^{N} \psi^{(s) T} \frac{\partial U\left(u, \lambda_{s}\right)}{\partial u} \phi^{(s)}\right)\right]\right)=\rho\left(\left[U_{0}, \sum_{s=1}^{N} \phi^{(s)} \psi^{(s) T}\right]\right) .
$$

On the other hand, it is easy to find that the multi-wave interaction equations (3.4) have a Lie point symmetry

$$
Y_{0}:=\rho\left(\left[\Gamma, U_{1}\right]\right), \Gamma=\operatorname{diag}\left(\gamma_{1}, \gamma_{2}, \gamma_{3}\right), \gamma_{i} \neq \gamma_{j}, 1 \leq i \neq j \leq 3
$$

where $\gamma_{1}, \gamma_{2}, \gamma_{3}$ are constants. Actually, we can directly prove that $Y_{0}$ and $Z_{0}$ are two symmetries of the multi-wave interaction equations (3.4). Set

$$
\delta U_{1}=\left[\Gamma, U_{1}\right] \text { or }\left[U_{0}, \sum_{s=1}^{N} \phi^{(s)} \psi^{(s) T}\right]
$$

then $\left[U_{0}, \delta V_{1}\right]=\left[V_{0}, \delta U_{1}\right]$ determines

$$
\delta V_{1}=\left[\Gamma, V_{1}\right] \text { or }\left[V_{0}, \sum_{s=1}^{N} \phi^{(s)} \psi^{(s) T}\right] .
$$

Now in view of (3.3), the symmetry problem requires to show that

$$
\left(\delta U_{1}, \delta V_{1}\right)=\left(\left[\Gamma, U_{1}\right],\left[\Gamma, V_{1}\right]\right) \text { or }\left(\left[U_{0}, \sum_{s=1}^{N} \phi^{(s)} \psi^{(s) T}\right],\left[V_{0}, \sum_{s=1}^{N} \phi^{(s)} \psi^{(s) T}\right]\right)
$$

satisfies the linearized system of the multi-wave interaction equations (3.4):

$$
\left(\delta U_{1}\right)_{t}-\left(\delta V_{1}\right)_{x}+\left[\delta U_{1}, V_{1}\right]+\left[U_{1}, \delta V_{1}\right]=0
$$


which just needs a direct computation.

Therefore, binary Bargmann symmetry constraint of (3.4) reads as

$$
Y_{0}=Z_{0} \text {, i.e., }\left[\Gamma, U_{1}\right]=\left[U_{0}, \sum_{s=1}^{N} \phi^{(s)} \psi^{(s) T}\right] .
$$

Solving this for $u$, we have the constraints on the potentials

$$
u_{i j}=\tilde{u}_{i j}:=\frac{\alpha_{i}-\alpha_{j}}{\gamma_{i}-\gamma_{j}}\left\langle\Phi_{i}, \Psi_{j}\right\rangle, 1 \leq i \neq j \leq 3
$$

where $\langle\cdot, \cdot\rangle$ denotes the standard inner-product of $\mathbb{R}^{N}$, and

$$
\Phi_{i}=\left(\phi_{i 1}, \cdots, \phi_{i N}\right)^{T}, \Psi_{i}=\left(\psi_{i 1}, \cdots, \psi_{i N}\right)^{T}, 1 \leq i \leq 3
$$

The substitution of $u$ with $\tilde{u}=\left(\tilde{u}_{21}, \tilde{u}_{12}, \tilde{u}_{31}, \tilde{u}_{13}, \tilde{u}_{23}, \tilde{u}_{32}\right)$ in binary Lax systems (4.4) and (4.5) leads to the so-called constrained flows:

$$
\begin{aligned}
& \phi_{x}^{(s)}=U\left(\tilde{u}, \lambda_{s}\right) \phi^{(s)}, \psi_{x}^{(s)}=-U^{T}\left(\tilde{u}, \lambda_{s}\right) \psi^{(s)}, 1 \leq s \leq N \\
& \phi_{t}^{(s)}=V\left(\tilde{u}, \lambda_{s}\right) \phi^{(s)}, \psi_{t}^{(s)}=-V^{T}\left(\tilde{u}, \lambda_{s}\right) \psi^{(s)}, 1 \leq s \leq N .
\end{aligned}
$$

These are two systems of ordinary differential equations. In the following two sections, we would like to show their Liouville integrability.

\section{Hamiltonian structures and Lax representations}

In order to prove that two constrained flows are Liouville integrable, we need generate their Hamiltonian structures and integrals of motion. First, the spatial constrained flow (4.15) can be easily expressed as the Hamiltonian form

$$
\Phi_{i x}=-\frac{\partial H^{x}}{\partial \Psi_{i}}, \Psi_{i x}=\frac{\partial H^{x}}{\partial \Phi_{i}}, 1 \leq i \leq 3
$$

with the Hamiltonian

$$
H^{x}=-\sum_{k=1}^{3} \alpha_{k}\left\langle A \Phi_{k}, \Psi_{k}\right\rangle-\sum_{1 \leq k<l \leq 3} \frac{\alpha_{k}-\alpha_{l}}{\beta_{k}-\beta_{l}}\left\langle\Phi_{k}, \Psi_{l}\right\rangle\left\langle\Phi_{l}, \Psi_{k}\right\rangle,
$$


where $A=\operatorname{diag}\left(\lambda_{1}, \lambda_{2}, \cdots, \lambda_{N}\right)$. Second, the temporal constrained flow (4.16) has a similar Hamiltonian structure

$$
\Phi_{i t}=-\frac{\partial H^{t}}{\partial \Psi_{i}}, \Psi_{i t}=\frac{\partial H^{t}}{\partial \Phi_{i}}, 1 \leq i \leq 3
$$

with the Hamiltonian

$$
H^{t}=-\sum_{k=1}^{3} \beta_{k}\left\langle A \Phi_{k}, \Psi_{k}\right\rangle-\sum_{1 \leq k<l \leq 3} \frac{\beta_{k}-\beta_{l}}{\gamma_{k}-\gamma_{l}}\left\langle\Phi_{k}, \Psi_{l}\right\rangle\left\langle\Phi_{l}, \Psi_{k}\right\rangle
$$

It is known that Lax representations are basic objects to generate integrals of motion. In order to present Lax representations of the constrained flows (4.15) and (4.16), let us define a Lax operator $L(\lambda)$ by

$$
L(\lambda)=\left(\begin{array}{ccc}
\gamma_{1}+\sum_{l=1}^{N} \frac{\phi_{1 l} \psi_{1 l}}{\lambda-\lambda_{l}} & \sum_{l=1}^{N} \frac{\phi_{1 l} \psi_{2 l}}{\lambda-\lambda_{l}} & \sum_{l=1}^{N} \frac{\phi_{1 l} \psi_{3 l}}{\lambda-\lambda_{l}} \\
\sum_{l=1}^{N} \frac{\phi_{2 l} \psi_{1 l}}{\lambda-\lambda_{l}} & \gamma_{2}+\sum_{l=1}^{N} \frac{\phi_{2 l} \psi_{2 l}}{\lambda-\lambda_{l}} & \sum_{l=1}^{N} \frac{\phi_{2 l} \psi_{3 l}}{\lambda-\lambda_{l}} \\
\sum_{l=1}^{N} \frac{\phi_{3 l} \psi_{1 l}}{\lambda-\lambda_{l}} & \sum_{l=1}^{N} \frac{\phi_{3 l} \psi_{2 l}}{\lambda-\lambda_{l}} & \gamma_{3}+\sum_{l=1}^{N} \frac{\phi_{3 l} \psi_{3 l}}{\lambda-\lambda_{l}}
\end{array}\right) .
$$

Now if we set $\widetilde{U}(\lambda)=U(\tilde{u}, \lambda)$ and $\widetilde{V}(\lambda)=V(\tilde{u}, \lambda)$, then it is easy to check the following Lax representations:

$$
(L(\lambda))_{x}=[\widetilde{U}(\lambda), L(\lambda)],(L(\lambda))_{t}=[\tilde{V}(\lambda), L(\lambda)]
$$

for the spatial constrained flow (4.15) and the temporal constrained flow (4.16), respectively. These Lax representations will be applied to construct the required integrals of motion for the Liouville integrability of two constrained flows in the next section.

\section{$6 r$-matrix formulation and Liouville integrability}

Let us specify the Poisson bracket:

$$
\{f, g\}=\sum_{i=1}^{3}\left(\left\langle\frac{\partial f}{\partial \Psi_{i}}, \frac{\partial g}{\partial \Phi_{i}}\right\rangle-\left\langle\frac{\partial f}{\partial \Phi_{i}}, \frac{\partial g}{\partial \Psi_{i}}\right\rangle\right), f, g \in C^{\infty}\left(\mathbb{R}^{6 N}\right) .
$$


An $r$-matrix formulation can be directly shown for the Lax operator $L(\lambda)$ defined by (5.5).

Theorem 6.1 The Lax operator $L(\lambda)$ given by (5.5) has the following $r$ matrix formulation

$$
\{L(\lambda) \stackrel{\otimes}{,} L(\mu)\}=\left[\frac{1}{\mu-\lambda} \mathcal{P}, L_{1}(\lambda)+L_{2}(\mu)\right], \mathcal{P}=\sum_{i, j=1}^{3} E_{i j} \otimes E_{j i},
$$

where $L_{1}(\lambda)=L(\lambda) \otimes I_{3}, L_{2}(\lambda)=I_{3} \otimes L(\mu)$, and

$$
\left(E_{i j}\right)_{k l}=\delta_{i k} \delta_{j l},\{L(\lambda) \stackrel{\otimes}{,} L(\mu)\}_{i j, k l}=\left\{(L(\lambda))_{i k},(L(\mu))_{j l}\right\}, 1 \leq i, j, k, l \leq 3 .
$$

First from the Lax representations in (5.6), we have

$$
\left(\nu I_{3}-L(\lambda)\right)_{x}=\left[\tilde{U}(\lambda), \nu I_{3}-L(\lambda)\right],\left(\nu I_{3}-L(\lambda)\right)_{t}=\left[\tilde{V}(\lambda), \nu I_{3}-L(\lambda)\right],
$$

where $\nu$ is a constant, and thus [14]

$$
\left(\operatorname{det}\left(\nu I_{3}-L(\lambda)\right)\right)_{x}=0,\left(\operatorname{det}\left(\nu I_{3}-L(\lambda)\right)\right)_{t}=0 .
$$

Second from the $r$-matrix formulation (6.2), we have [15]

$$
\left\{\operatorname{tr} L^{k}(\lambda), \operatorname{tr} L^{l}(\mu)\right\}=0, k, l \geq 1 .
$$

Expand the determinant of the matrix $\nu I_{3}-L(\lambda)$ as

$$
\operatorname{det}\left(\nu I_{3}-L(\lambda)\right)=\nu^{3}-\mathcal{F}_{1}(\lambda) \nu^{2}+\mathcal{F}_{2}(\lambda) \nu-\mathcal{F}_{3}(\lambda),
$$

where by Newton's identities on elementary symmetric polynomials,

$$
\begin{aligned}
& \mathcal{F}_{1}(\lambda)=\operatorname{tr} L(\lambda), \mathcal{F}_{2}(\lambda)=\frac{1}{2}\left((\operatorname{tr} L(\lambda))^{2}-\operatorname{tr} L^{2}(\lambda)\right), \\
& \mathcal{F}_{3}(\lambda)=\frac{1}{6}(\operatorname{tr} L(\lambda))^{3}+\frac{1}{3} \operatorname{tr} L^{3}(\lambda)-\frac{1}{2}(\operatorname{tr} L(\lambda)) \operatorname{tr} L^{2}(\lambda) .
\end{aligned}
$$

Thus, it follows that

$$
\left(\mathcal{F}_{i}(\lambda)\right)_{x}=0,\left(\mathcal{F}_{i}(\lambda)\right)_{t}=0, \text { and }\left\{\mathcal{F}_{i}(\lambda), \mathcal{F}_{j}(\mu)\right\}=0,1 \leq i, j \leq 3 .
$$

Further expand $\mathcal{F}_{i}(\lambda)$ as

$$
\mathcal{F}_{i}(\lambda)=\sum_{l \geq 0} F_{i l} \lambda^{-l}, 1 \leq i \leq 3,
$$


where obviously the $F_{i 0}$ 's are constants. Then from the last equality in (6.4), we have

$$
\left\{F_{i l}, F_{j k}\right\}=0,1 \leq i, j \leq 3, k, l \geq 0
$$

The first two equalities in (6.4) and the equality (6.6) implies that two constrained flows (4.15) and (4.16) have the common involutive integrals of motion: $F_{i l}, \quad 1 \leq i \leq 3, l \geq 1$. Now it is a direct computation to verify the following theorem on the Liouville integrability of the constrained flows (4.15) and (4.16).

Theorem 6.2 Two constrained flows (4.15) and (4.16) have the common involutive integrals of motion: $F_{i l}, 1 \leq i \leq 3, l \geq 1$, of which the functions $F_{\text {is }}, 1 \leq i \leq 3,1 \leq s \leq N$, are functionally independent over a dense open subset of $\mathbb{R}^{6 N}$. Therefore, the constrained flows (4.15) and (4.16) are Liouville integrable.

\section{$7 \quad$ Involutive solutions}

Since under the constraints on the potentials (4.13), the compatability condition of (4.4) and (4.5) is still the multi-wave interaction equations (3.4), solutions $\left(\Phi_{i}(x, t), \Psi_{i}(x, t)\right)$ to the constrained flows (4.15) and (4.16) present solutions to the multi-wave interaction equations (3.4):

$$
u_{i j}(x, t)=\frac{\alpha_{i}-\alpha_{j}}{\gamma_{i}-\gamma_{j}}\left\langle\Phi_{i}(x, t), \Psi_{j}(x, t)\right\rangle, 1 \leq i \neq j \leq 3
$$

This also shows the integrability by quadratures for the multi-wave interaction equations (3.4) since $\Phi_{i}$ and $\Psi_{i}$ can be determined by quadratures. On the other hand, it is easily found that under the Poisson bracket (6.1), two Hamiltonians $H^{x}$ and $H^{t}$ commute, i.e.,

$$
\left\{H^{x}, H^{t}\right\}=\sum_{i=1}^{3}\left(\left\langle\frac{\partial H^{x}}{\partial \Psi_{i}}, \frac{\partial H^{t}}{\partial \Phi_{i}}\right\rangle-\left\langle\frac{\partial H^{x}}{\partial \Phi_{i}}, \frac{\partial H^{t}}{\partial \Psi_{i}}\right\rangle\right)=0
$$

Hence, the above solutions given by (7.1) determine involutive solutions to the multi-wave interaction equations (3.4). If we denote two Hamiltonian flows of (4.15) and (4.16) by $g_{x}^{H^{x}}$ and $g_{t}^{H^{t}}$ respectively, then we have

$$
\begin{aligned}
u_{i j}(x, t) & =\frac{\alpha_{i}-\alpha_{j}}{\gamma_{i}-\gamma_{j}}\left\langle g_{x}^{H^{x}} g_{t}^{H^{t}} \Phi_{i 0}, g_{x}^{H^{x}} g_{t}^{H^{t}} \Psi_{j 0}\right\rangle \\
& =\frac{\alpha_{i}-\alpha_{j}}{\gamma_{i}-\gamma_{j}}\left\langle g_{t}^{H^{t}} g_{x}^{H^{x}} \Phi_{i 0}, g_{t}^{H^{t}} g_{x}^{H^{x}} \Psi_{j 0}\right\rangle, \quad 1 \leq i \neq j \leq 3
\end{aligned}
$$


where the initial values $\Phi_{i 0}$ and $\Psi_{i 0}$ of $\Phi_{i}$ and $\Psi_{i}$ can be taken as any arbitrary constant vectors of $\mathbb{R}^{N}$.

Summing up, solutions of the constrained flows (4.15) and (4.16) lead to involutive solutions of the multi-wave interaction equations (3.4). Moreover, such involutive solutions show us (a) the richness of solutions of the multiwave interaction equations (3.4), and (b) the integrability by quadratures for the multi-wave interaction equations (3.4).

\section{Summary and conclusion}

Binary Bargmann symmetry constraints in the continuous case decompose soliton equations (PDEs) into constrained flows (ODEs). The $r$-matrix formulation can be used to show the Liouville integrability of the constrained flows. The resulting constraints on the potentials give rise to involutive solutions to soliton equations and thus show the integrability by quadratures for soliton equations. The whole process of binary Bargmann symmetry constraints, called binary nonlinearization, can be depicted as follows.

\begin{tabular}{|c|c|c|}
\hline $\begin{array}{l}\text { Lax system and } \\
\text { adjoint Lax system }\end{array}$ & $\underset{\text { symmetry }}{\stackrel{\text { by }}{\longrightarrow}}$ & $\begin{array}{l}\text { Constrained spatial } \\
\text { and temporal flows }\end{array}$ \\
\hline $\begin{array}{l}\left\{\begin{array}{l}\phi_{x}=U(u, \lambda) \phi \\
\phi_{t}=V(u, \lambda) \phi\end{array}\right. \\
\left\{\begin{array}{l}\psi_{x}=-U^{T}(u, \lambda) \psi \\
\psi_{t}=-V^{T}(u, \lambda) \psi\end{array}\right.\end{array}$ & & $\begin{aligned} \phi_{x} & =U(\tilde{u}(\phi, \psi), \lambda) \phi \\
\psi_{x} & =-U^{T}(\tilde{u}(\phi, \psi), \lambda) \psi \\
\phi_{t} & =V(\tilde{u}(\phi, \psi), \lambda) \phi \\
\psi_{t} & =-V^{T}(\tilde{u}(\phi, \psi), \lambda) \psi \\
& \downarrow \begin{array}{l}\text { by } r \text {-matrix } \\
\text { formulation }\end{array}\end{aligned}$ \\
\hline $\begin{array}{c}\text { Integrability by } \\
\text { quadratures for } \\
U_{t}-V_{x}+[U, V]=0\end{array}$ & 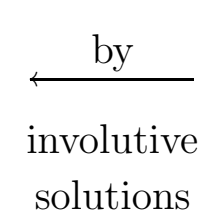 & $\begin{array}{c}\text { finite-dimensional } \\
\text { Liouville integrable } \\
\text { Hamiltonian systems }\end{array}$ \\
\hline
\end{tabular}

The multi-wave interaction equations (3.4) have been taken as an illustrative example. Binary Bargmann symmetry constraints (4.12), containing a 
set of arbitrary distinct constants $\gamma_{1}, \gamma_{2}, \gamma_{3}$, were proposed for the multi-wave interaction equations (3.4). Two finite-dimensional Liouville integrable Hamiltonian systems (5.1) and (5.3) resulted from the constrained flows determine involutive solutions to the multi-wave interaction equations (3.4). Our result with a special case $\Gamma=V_{0}$, i.e., $\operatorname{diag}\left(\gamma_{1}, \gamma_{2}, \gamma_{3}\right)=\operatorname{diag}\left(\beta_{1}, \beta_{2}, \beta_{3}\right)$, gives rise to all the results established in [16].

Of special interest in the study of binary symmetry constraints are to create new classical integrable Hamiltonian systems which supplement the known classes of classical integrable systems [17] and to expose the integrability by quadratures for soliton equations by using constrained flows [9]. We point out that high-order symmetry constraints with involved symmetries having degenerate Hamiltonians need particular consideration [18]. A profound mathematical theory on binary symmetry constraints, especially on Hamiltonian structures of constrained flows, will be discussed elsewhere.

\section{Acknowledgments}

This work was supported by a grant from the Research Grants Council of Hong Kong Special Administrative Region, China (Project no. 9040466), and a grant from the City University of Hong Kong (Project no. 7001041). The author is also grateful to Prof. Z. X. Zhou for stimulating discussions.

\section{References}

[1] C. W. Cao, Nonlinearization of the Lax system for AKNS hierarchy, Sci. China Ser. A 33 (1990) 528-536.

[2] C. W. Cao and G. X. Geng, Classical integrable systems generated through nonlinearization of eigenvalue problems, In: C. H. Gu, Y. S. Li and G. Z. Tu, eds., Nonlinear Physics, Proceedings, Shanghai, 1989, (Springer-Verlag, Berlin, 1990) 68-78.

[3] Y. B. Zeng and Y. S. Li, Three kinds of constraints of potential for KdV hierarchy, Acta Math. Sinica (N.S.) 6 (1990) 257-272.

[4] W. X. Ma and W. Strampp, An explicit symmetry constraint for the Lax pairs and the adjoint Lax pairs of AKNS systems, Phys. Lett. A 185 (1994) 277-286.

[5] W. X. Ma, Symmetry constraint of MKdV equations by binary nonlinearization, Physica A 219 (1995) 467-481.

[6] W. X. Ma, B. Fuchssteiner and W. Oevel, A $3 \times 3$ matrix spectral problem for AKNS hierarchy and its binary nonlinearization, Physica A 233 (1996) 331-354. 
[7] J. C. Eilbeck, V. Z. Enolskii, V. B. Kuznetsov and A. V. Tsiganov, Linear $r$-matrix algebra for classical separable systems, J. Phys. A: Math. Gen. 27 (1994) 567-578.

[8] W. X. Ma and Y. B. Zeng, Binary constrained flows and separation of variables for soliton equations, to appear in Proceedings of the Conference on Integrable Systems in celebration of Martin D. Kruskal's 75th Birthday, Adelaide, Australia, 2000.

[9] W. X. Ma and B. Fuchssteiner, Binary nonlinearization of Lax pairs, In: E. Alfinito, M. Boiti, L. Martina and F. Pempinelli, eds., Nonlinear Physics: Theory and Experiment, Proceedings, Lecce, 1995 (World Sci. Publishing, River Edge, NJ, 1996) 217-224.

[10] W. X. Ma and X. G. Geng, Bäcklund transformations of soliton systems from symmetry constraints, to appear in Proceedings of the AARMS-CRM Workshop on Bäcklund \& Darboux Transformations: The Geometry of Soliton Theory, Halifax, Canada, 1999.

[11] W. X. Ma, Binary nonlinearization for the Dirac systems, Chinese Ann. Math. Ser. B 18 (1997) 79-88.

[12] V. E. Zakharov and S. V. Manakov, Resonant interaction of wave packets in nonlinear media, Sov. Phys. JETP Lett. 18 (1973) 243-245.

[13] D. J. Kaup, The three-wave interaction - a nondispersive phenomenon, Stud. Appl. Math. 55 (1976) 9-44.

[14] G. Z. Tu, On Liouville integrability of zero-curvature equations and the Yang hierarchy, J. Phys. A: Math. Gen. 22 (1989) 2375-2392.

[15] O. Babelon and C. M. Viallet, Hamiltonian structures and Lax equations, Phys. Lett. B 237 (1990) 411-416.

[16] Y. T. Wu and G. X. Geng, A finite-dimensional integrable system associated with the three-wave interaction equations, J. Math. Phys. 40 (1999) 3409-3430.

[17] A. M. Perelomov, Integrable systems of classical mechanics and Lie algebras, Vol. I. (Birkhäuser Verlag, Basel, 1990).

[18] Y. S. Li and W. X. Ma, Binary nonlinearization of AKNS spectral problem under higher-order symmetry constraints, Chaos, Solitons \& Fractals 11 (2000) 697-710. 